\title{
COLLECTIVE DECISION-MAKING BY A GROUP OF COCKROACH-LIKE ROBOTS *
}

\author{
Simon Garnier ${ }^{\dagger a}$, Christian Jost ${ }^{a}$, Raphaël Jeanson ${ }^{a}$, Jacques Gautrais ${ }^{a}$, \\ Masoud Asadpour ${ }^{b}$, Gilles Caprari ${ }^{b}$ and Guy Theraulaz ${ }^{a}$ \\ ${ }^{a}$ Centre de Recherches sur la Cognition Animale \\ UMR-CNRS 5169, Bât 4R3, Université Paul Sabatier \\ 118 Route de Narbonne - F-31062 Toulouse cedex 4 - FRANCE \\ ${ }^{b}$ Autonomous Systems Lab \\ Swiss Federal Institute of Technology (EPFL) \\ CH-1015 Lausanne, SWITZERLAND
}

\begin{abstract}
In group-living animals, aggregation favours interactions as well as information exchanges between individuals, and allows thus the emergence of complex collective behaviors. In previous works, a model of a self-enhanced aggregation was deduced from experiments with the cockroach Blattella germanica. In this work, this model was implemented in micro-robots Alice and successfully reproduced the agregation dynamics observed in a group of cockroaches. We showed that this aggregation process, based on a small set of simple behavioral rules and interactions among individuals, can be used by the group of robots to select collectively an aggregation site among two identical or different shelters. Moreover, we showed that the aggregation mechanism allows the robots as a group to "estimate" the size of each shelter during the collective decision-making process, a capacity which is not explicitly coded at the individual level but that simply emerges from the aggregation behaviour.
\end{abstract}

\section{INTRODUCTION}

Since the last 15 years, collective robotics has undergone a considerable development [27]. In order to control the behavior of a group of robots, collective robotics was often inspired by the collective abilities demonstrated by social insects $[4,24]$. Indeed, nature has already developed many strategies that solve collective problems through the decentralized organisation and coordination of many autonomous agents by self-organized mechanisms [6] (for instance trail

\footnotetext{
* This work was partly supported by a European community grant given to the LEURRE project under the "Information Society Technologies" Programme (2002-2005), contract FET-OPEN-IST-2001-35506 of the Future and Emerging Technologies arm and by the Programme Cognitique from the French Ministry of Scientific Research. The authors would like to thank Jean-Louis Deneubourg for all its very helpful advices about this work.

${ }^{\dagger}$ Corresponding author. simon.garnierdcict. fr
}

formation [21], food source selection [25], division of labor [5] or collective defense [20]).

Among all these self-organized behaviours, aggregation is one of the simplest. But it is also one of the most useful. For instance, it allows an individual to transmit an information in a very efficient way to many other conspecifics at the same time. It thus favours recruitment processes during food source exploitation [6] or territory defense [9]. Aggregation also facilitates the interactions among individuals, leading to complex collective behaviors such as nest construction [11], nest-site selection [16] or traffic regulation [10]. To sum up, aggregation is a step toward much more complex collective behaviours because it favours interactions and information exchanges among individuals, leading to the emergence of complex and functional self-organized structures. As such it plays a keyrole in the evolution of cooperation in animal societies [8].

Such self-organized aggregation processes were regularly used in collective robotics. For instance, foraging tasks (i.e. clustering of objects scattered in the environment) were used to study the impact of the group size [19] or of a simple form of communication [26] on the harvest efficiency. But even more complex consequences of aggregation processes were studied with groups of robots. For instance, Agassounon and Martinoli [1] showed that division of labor can emerge in a group of foraging robots when the size of the group grows. Beckers et al. [3] and Holland and Melhuish [14] showed that an object clustering paradigm based on stigmergy [13] can lead a group of robots to order and assemble objects of two different types.

In this paper we address a new collective behavior that is based on self-organized aggregation of robots themselves. We show that a self-enhanced aggregation process, which leads groups of cockroaches to a quick and strong aggregation [17], can be used by a group of mini-robots Alice 
to select collectively an aggregation site among two identical or different shelters. We show that, even though these robots have limited sensory and cognitive abilities, they are still able to perform a collective decision. It has already been shown that such self-enhanced mechanisms are used by insects to make collective decisions: for instance in food source selection in bees [25], in nest site selection in ants [16] or in resting site selection in cockroaches [2]. This collective choice appears each time through the amplification of small fluctuations in the use of two (or more) targets. These fluctuations arise from behavioral randomness and/or from natural preferences of animals (in the case of different targets) and are amplified by recruitment processes (through pheromone deposits for instance) [6].

Here we propose that a biological model of aggregation of first instar larvae of the cockroach Blattella germanica $[15,17]$ can lead a group of robots Alice to the collective choice of a "resting" site (or shelter). We show that a very simple self-enhanced mechanism underlying this aggregation process is sufficient to make the group of robots aggregate under one of two identical shelters, instead of equally splitting between them. If the two shelters are different (here in size), we also show that robots preferentially choose the biggest of the two, without being individually able to measure the size of each shelter.

In this paper, we first describe the biological model of aggregation we have used and the way this model was implemented in a group of mini-robots Alice. We then show that this implementation indeed results in a collective aggregation behavior that is quantitatively indistinguishable from cockroach aggregation. Finally, we show that, when this aggregation behavior is restricted to certain zones in the environment (for instance by natural preferences for dark places as in cockroaches [23]), the robots preferentially aggregate in only one of these zones, i.e. they collectively choose a single "resting" site. The results of our experiments were also used to calibrate a computer simulation of robots Alice that will allow us to extend the exploration of this collective decision model in further studies.

\section{SELF-ORGANIZED AGGREGATION}

2

The aggregation process cited above is directly inspired by a biological model of displacement and aggregation developed from experiments with first instar larvae of the german cockroach Blattella germanica [15, 17]. This model was built by quantifying individual behaviors of cockroaches, that is their displacement, interactions among individuals and with the environment in a homogeneous circular arena (11 cm diameter) . Each of these individual behaviors was described in a probabilistic way: we measured experimentally the probability distribution for a given behavior to hap- pen.

This analysis showed that cockroaches display a correlated random walk (constant rate to change direction and forward oriented distribution of turning angles) in the center of the arena [15]. When reaching the periphery of the arena, cockroaches display a wall following behavior (also called thigmotactic behavior) with a constant rate to leave the edge and come back into the central part of the arena [15]. In addition, cockroaches can stop at any moment, stay motionless for some time and then move again. Analysis showed that the stopping rate for an individual increases with the number of stopped cockroaches in the direct neighbourhood (i.e. within the range of antenna contact) [17]. On the contrary, the rate to leave an aggregate decreases with this number [17]. Thus, this dual positive feedback leads to the quick and strong formation of aggregates (as can be seen in Fig. 1). A more detailed description of the model can be found in Jeanson et al. $[15,17]$.

The first part of our work was to implement this biological model of aggregation in the micro-robots Alice. These robots were designed at the EPFL (Lausanne, Switzerland) [7]. They are very small robots $(22 \mathrm{~mm} \times 21 \mathrm{~mm} \times 20 \mathrm{~mm})$ equipped with two watch motors with wheels and tires allowing a maximum speed of $40 \mathrm{~mm} \mathrm{~s}^{-1}$. Four infra-red sensors are used for obstacle detection and local communication among Alices (up to $4 \mathrm{~cm}$ distance). Robots have a microcontroller PIC16LF877 with 8K Flash EEPROM memory, 368 bytes RAM but no built-in float operations. To determine the number of neighbors (upon which relies of the aggregation process), each robot owns a specific identification number and counts the number of nearby neighbors in a distance roughly less than $4 \mathrm{~cm}$ ) with a different id number. Intrinsic differences between the perception area of robots and cockroaches and imperfect neighbor counts due to noise in IR devices required some fine-tuning of the behavioral parameters in order for the behavioural output of the robots to correctly match the cockroach individual behaviors. This behavioral output of robots was measured using the same experimental methods (10 to 30 experiments depending on the studied behavior) as those used to characterize the individual behavior of cockroaches (see Jeanson et al. [15, 17] for a detailed description of these methods).

However individual behaviors are not yet aggregation behavior, and the true validation of the model implementation must be done at the collective level by comparing the aggregation behavior of robots to the aggregation behavior of cockroaches. To this aim, we ran the following aggregation experiment: groups of robots (10 or 20 individuals) were put into a homogeneous white circular arena $(50 \mathrm{~cm}$ diameter) during 60 minutes. This experiment is similar to the one done by Jeanson et al. [17] with cockroaches. 
To draw a parallel between cockroach aggregation behavior and robot aggregation behavior, we scaled the dimensions of the robot arena so that it matches scale differences between robot and cockroach sizes. The experiment was repeated 10 times for each group size (10 or 20 robots). The aggregation dynamics were characterized through three kinds of measurements (sampled every minute): size of the largest aggregate, number of aggregates and number of isolated individuals (see Jeanson et al. $[15,17]$ for a detailed description of these measurements). For each of these three dynamics, the experimental results showed a very good agreement between robots and cockroaches, confirming that the cockroach aggregation process was well implemented in the robots Alice (see Fig. 1).

\section{COLLECTIVE CHOICE}

This aggregation process implemented in robots can occur in the whole experimental arena, without any preference for a given location. Actually, in nature some places are more attractive for cockroaches, thus promoting aggregation in particular sites. For instance, cockroaches preferentially aggregate in dark places [23]. Experimentally, if one puts a dark shelter in a lighted arena (as the one used for the study of cockroach aggregation), one can observe that cockroaches strongly aggregate under this shelter. And if two or more dark shelters are placed in the arena, one can observe that a majority of cockroaches aggregates under only one of these shelters, rather than evenly spreading their population among all the aggregation sites [18]. Thus cockroaches are able to perform a collective choice for a given aggregation site, even if these sites are identical.

Though the mechanisms leading to this collective choice are not yet fully understood, we suggest that this choice could strongly rely on the self-enhanced aggregation process described above and tested with robots. Indeed such self-enhanced mechanisms are already known to lead animal groups to collective decisions, such as the collective choice of a food source [25] and of a target [20] in bees, of a nest site in ants [16], or of a resting site in cockroaches [2]. In this latter case, Ame et al. [2] showed that the simple modulation of the resting period on a given site by the number of individuals on that site leads the group of cockroaches to the choice of one shelter among two or more identical ones. We argue that this modulation can be achieved easily through the aggregation process described above. To test our hypothesis, we ran three sets of experiments during which a group of robots was faced to the choice between two potential aggregation sites. Besides proving that collective decision in robots can appear from a simple aggregation process, these experiments were also used to calibrate a simulation tool which will be used in further studies to identify the behavioral parameters that control collective choice (see
Fig. 2 for some pictures of both experiments wih robots and simulations).

The first set of experiments was designed to ascertain whether the cockroach aggregation behavior is able to lead a group of robots to a collective choice between two identical targets. To that aim, we put a group of 10 robots in the same arena as the one used for aggregation experiments, except that we added just above the arena two dark shelters. These shelters were of the same size $(14 \mathrm{~cm}$ diameter) and each of them can house the whole population of

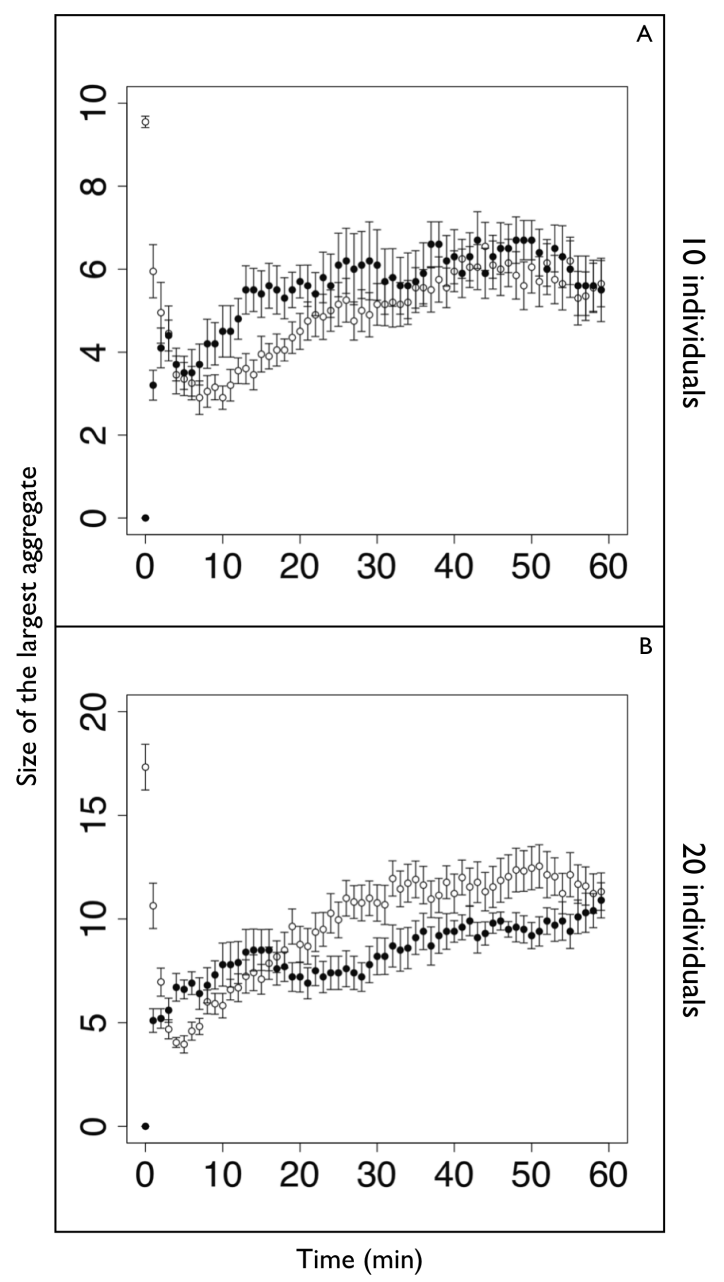

Figure 1. Aggregation dynamics: size of the largest aggregate. A: experiments with 10 individuals. B: experiments with 20 individuals. Black dots represent data for robots; white dots represent data for cockroaches. Each dot represents the mean \pm standard error (s.e.). Initial differences between starting points of robot and cockroach dynamics are solely due to the way cockroaches have to be brought into the arena as explained in Jeanson et al. [17]. 


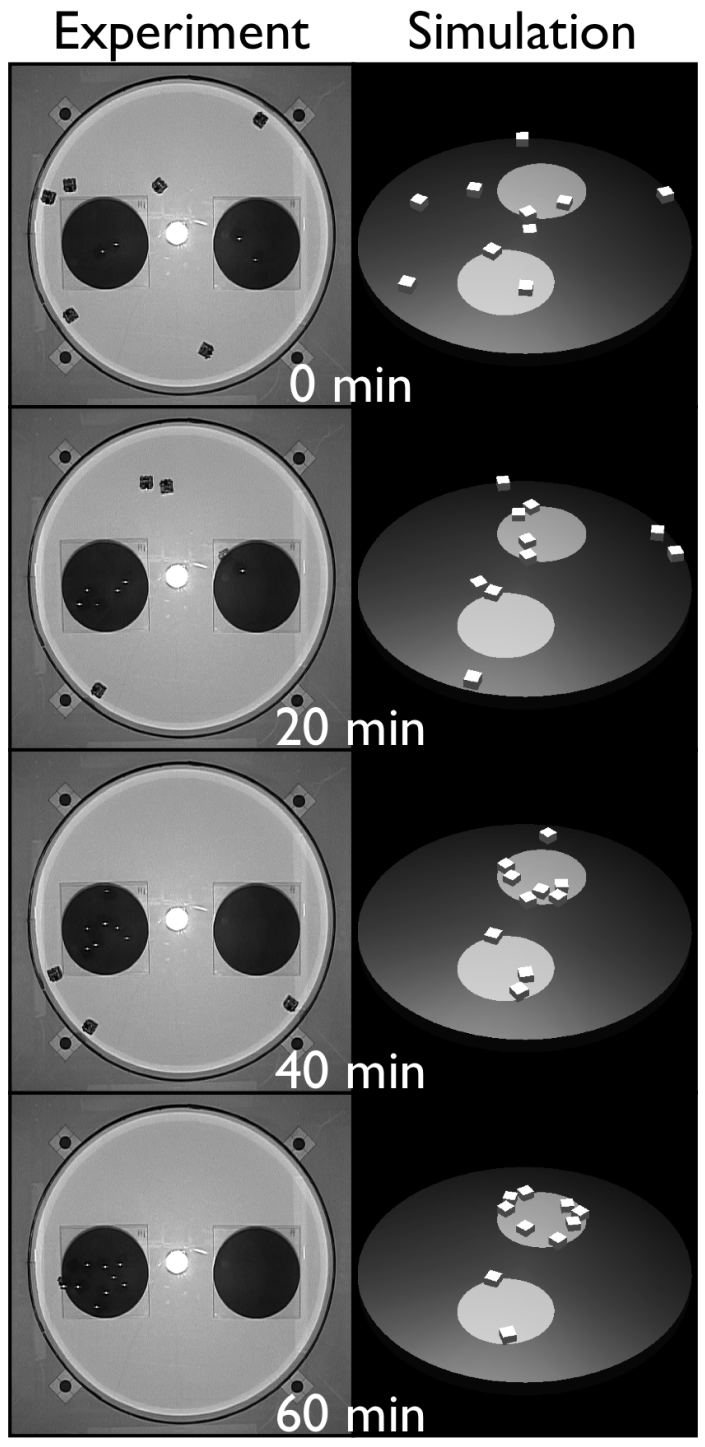

FigURE 2. Snapshots of an experiment (left) and a simulation (right) taken every 20 minutes during 60 minutes. These snapshots corresponds to the experiment with two identical shelters (14 $\mathrm{cm}$ diameter). As can be seen, the experiment ended with the choice of one of the two shelters by both real and simulated robots.

robots. Robots used the same behavioral algorithm as the one previously tested for its aggregation ability, except that, now, robots only stop under dark shelters (that is when IR light intensity falls under a given threshold). 20 experiments were performed, each lasting 60 minutes.

The number of stopped robots under each shelter was measured every minute to characterize the aggregation dynamics under each shelter. In addition, we also computed the percentage of stopped robots under each shelter at the end of each experiment to characterize the collective choice of the group of robots. From this last measurement, we derive what we call a "choice distribution". For a given shelter, this choice distribution corresponds to the number of experiments ending with a given percentage of stopped robots under this shelter (the choice distribution being symmetrical for the other shelter). For instance, how many experiments ended with 0 to 20 percent of stopped robots under shelter number 1 ? Or with 20 to 40 percent, etc. Note that a robot can be in one of the three locations at the end of an experiment: under shelter 1, under shelter 2 or outside the shelters. In the case of each robot choosing randomly a shelter (i.e. without any influence of its conspecifics), the result will follow a trinomial law with parameters $m_{t o t}=10$ (number of robots), $p_{a}=\left(m_{t o t}-m_{s}\right) / m t o t$ ( $p_{a}$, probability for a robot to be outside the shelters; $m_{s}$ number of robots stopped under any shelter, parametrized from the experiments), $p_{s 1}=\left(1-p_{a}\right)\left(r_{s 1}^{2} /\left(r_{s 1}^{2}+r_{s 2}^{2}\right)\right)\left(p_{s 1}\right.$, probability for a robot to be under shelter $1 ; r_{s 1}$, radius of shelter 1 ; $r_{s 2}$, radius of shelter 2$)$ and $p_{s 2}=1-p_{s 1}-p_{a}\left(p_{s 2}\right.$, probability for a robot to be under shelter 2). The choice distribution resulting from this trinomial law can be obtained through Monte Carlo simulations (10000 simulations of 20 replicates). In the case of identical shelters, this choice distribution displays a centered peak as can be seen in Fig. 3 B.1, meaning that a majority of experiments ended with no choice for a particular shelter.

Contrary to the trinomial resulting choice distribution, the choice distribution obtained in experiments with two identical shelters displays two peaks, one at each side (see Fig. 3 B.2). A chi square test shows a strong difference between the trinomial and experimental distributions $\left(\chi^{2}=\right.$ $367.6885, d f=4, p<0.0001)$. Similar results are obtained with simulations (see Fig. 3 B.3) and a chi square analysis of contingency tables shows no difference between experiments and simulations $\left(\chi^{2}=2.1007, p=0.7322\right.$, $\mathrm{p}$-value simulated with 10000 replicates [22]). This U-shape distribution corresponds to two different "populations of experiments", each of them preferentially ending with the choice of a given shelter. Furthermore, in this case with two identical shelters, the symmetry of the U-shape means that each shelter is randomly chosen from one experiment to another. The dynamics of this choice can be seen in Figs. 4 B.1 and B.2. It shows that the choice occurs very rapidly within the first minutes of the experiments. It also shows that this choice is very strong, since $75.5 \pm 3.36 \%$ (mean \pm s.e., $n=$ 20 ) of the population of robots is under the chosen shelter at the end of the experiments $(78 \pm 0.53 \%, n=1000$, in simulations). Thus this set of experiments clearly shows that the aggregation process described above (with very simple individual behaviors) can lead a group of robots to perform a collective choice between two aggregation sites. 


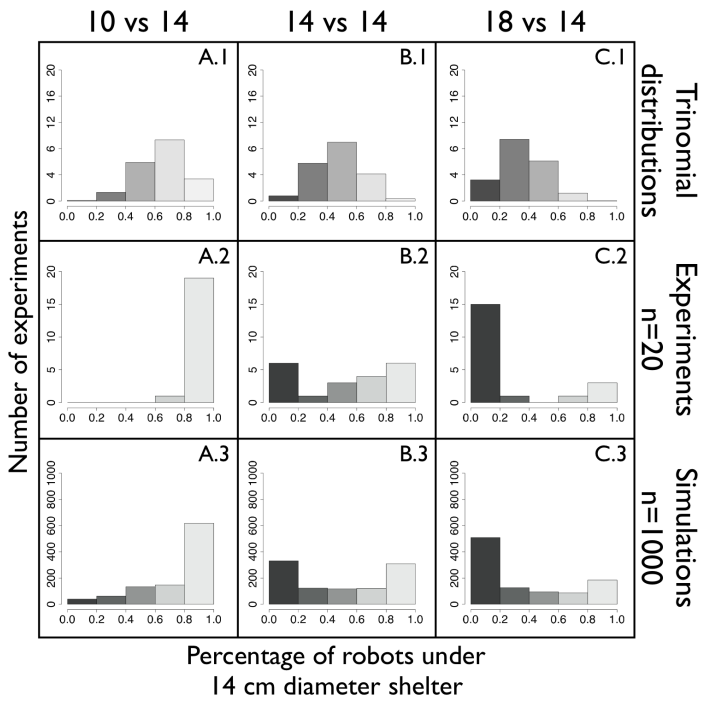

Figure 3. Choice distributions. In these distributions, each block represents a number of experiments ending with a given percentage $(0-20,20-40,40-60,60-80$ and 80-100 percent) of robots under one of the two shelters. Top: trinomial distributions (random choice). Middle: experimental distributions ( $n=20)$. Bottom: simulation distributions $(n=1000)$. Columns $\mathbf{A}$ and $\mathbf{C}$ represent choice distributions for the $14 \mathrm{~cm}$ diameter shelter against either the $10 \mathrm{~cm}$ diameter shelter (column A) or the $18 \mathrm{~cm}$ diameter shelter (column C). For each of these distributions, blocks on the right mean choice of the $14 \mathrm{~cm}$ diameter shelter and blocks on the left mean choice of the other shelter (either 10 or 18 $\mathrm{cm}$ diameter). Column $\mathbf{B}$ represents the choice distribution for a $14 \mathrm{~cm}$ diameter shelter against an other $14 \mathrm{~cm}$ shelter.

The two other sets of experiments were designed to assess the impact of a qualitative difference between the two shelters on the collective choice. As in the previous set of experiments, a group of 10 robots faced a choice between two shelters. But this time, while one of the shelters kept the same size as in the previous experiment, the size of the other was altered.

In a first set of 20 experiments, we confronted a $14 \mathrm{~cm}$ diameter shelter (able to house the whole robot population) with a $10 \mathrm{~cm}$ diameter shelter (too small to house the whole population of robots). As can be seen in Figs. 4 A.1 and A.2, robots quickly and strongly choose the shelter able to house their whole population. Thus, at the end of the experiments, $68 \pm 3.29 \%$ (mean \pm s.e., $n=20$ ) of the population is under the $14 \mathrm{~cm}$ diameter shelter $(72.7 \pm 0.79 \%$, $n=1000$, in simulations). The choice distribution shows a strong shift towards the $14 \mathrm{~cm}$ diameter shelter (see Fig. 3 A.2). This shift is the result of more than the simple difference between the area of the two shelters. Indeed, a comparison between the experimental distribution and a trinomial

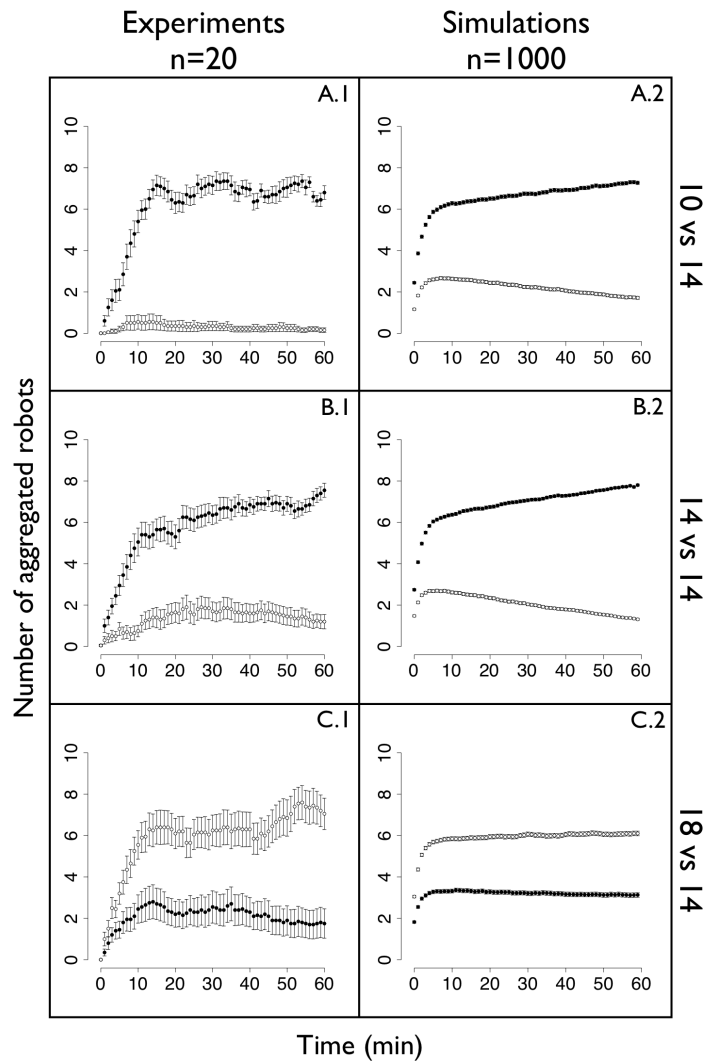

FIGURE 4. Choice dynamics: number of robots aggregated under each shelter. Top: experimental data $(n=20)$. Bottom: simulation data $(n=1000)$. In column $\mathbf{A}$ and $\mathbf{C}$, black dots represent data for the $14 \mathrm{~cm}$ diameter shelter; white dots represents data for either the $10 \mathrm{~cm}$ diameter shelter (column A) or the $18 \mathrm{~cm}$ diameter shelter (column C). In column B, black dots represent data for the chosen shelter (i.e. the shelter which is chosen at the end of each experiment); white dots represent data for the "not chosen" shelter. In all cases, each dot represents the mean \pm s.e.

distribution (Fig. 3 A.1) taking into account this difference in size shows a strong difference $\left(\chi^{2}=365.3578, d f=4\right.$, $p<0.0001$ ). Similar results are obtained with simulations (see Fig. 3 A.3) and a chi square analysis of contingency tables shows no difference between experiments and simulations $\left(\chi^{2}=9.4322, p=0.0595\right.$, $\mathrm{p}$-value simulated with 10000 replicates [22]). The disappearance of the U-shape of the distribution means that it remains only one "population of experiments" preferentially ending with the choice of the $14 \mathrm{~cm}$ diameter shelter, i.e. the one able to house the whole population of robots.

In a second set of 20 experiments, we confronted a 14 $\mathrm{cm}$ diameter shelter with a $18 \mathrm{~cm}$ diameter shelter. Both shelters are able to house the whole population of robots. 
As can be seen in Figs. 4 C.1 and C.2, robots choose the $18 \mathrm{~cm}$ diameter shelter. Thus, at the end of the experiments, $70.5 \pm 7.56 \%$ (mean \pm s.e., $n=20$ ) of the population is under the $14 \mathrm{~cm}$ diameter shelter $(61 \pm 1.12 \%, n=1000$, in simulations). The choice distribution shows a shift towards the $18 \mathrm{~cm}$ diameter shelter (see Fig. 3 C.2). This shift is the result of more than the simple difference between the area of the two shelters. Indeed, a comparison between the experimental distribution and a trinomial distribution (Fig. 3 C.1) taking into account this difference in size shows a strong difference $\left(\chi^{2}=373.8106, d f=4, p<0.0001\right)$. Similar results are obtained with simulations (see Fig. 3 C.3) and a chi square analysis of contingency tables shows no difference between experiments and simulations $\left(\chi^{2}=5.4480\right.$, $p=0.2301, \mathrm{p}$-value simulated with 10000 replicates [22]). But contrary of the previous experiment, the U-shape of the distribution has not disappeared and the two "populations of experiments" still exist: one that preferentially ended by a choice of the $14 \mathrm{~cm}$ diameter shelter, the other that preferentially ended by a choice of the $18 \mathrm{~cm}$ diameter shelter, the latter prevailing on the former.

From the two latter sets of experiments, we can conclude that the group of robots will choose preferentially a shelter that is sufficiently large to house all its members. But when the group is confronted with two sufficiently large shelters, the self-enhanced aggregation mechanism can lead the group to two stable choices, with a preference for the larger shelter. This implies that the group of robots is able to "sense" and "compare" the size of the shelters during the collective decision process, a performance that is beyond the direct scope of the simple aggregation process used in these experiments and that is not explicitly implemented in individual robots.

\section{CONCLUSION}

In this work, we achieved a collective decision process from a simple biological model of aggregation. We showed that a self-enhanced aggregation process associated with a preference for a given type of environmental heterogeneity (here a preference for dark places) can lead a group of robots to a collective choice for an aggregation site. Furthermore, this choice can be related to a collective ability to "sense" and "compare" the sizes of the aggregation sites. The most interesting aspect is that individual robots are unable to perform such behaviors (sensing the size of the shelters and choosing one of them) because of their very limited perception apparatus and computing power, and also because of the simplicity of their individual behaviors. But, as it has already been shown in insects $[2,6,10,20,25]$ and robots $[1$, 14], this simplicity is not a limit to the appearance of complex collective behavior. Division of labor [1], object ordering [14] and even collective decisions can emerge from the numerous interactions between artificial agents with some very simple behavioral rules.

To explain the ability of the group to choose preferentially the biggest shelter in our experiments, we hypothesise that this relies on the higher probability for the robots to encounter this shelter in the arena. Indeed, the more robots encounter a shelter, the more likely they will stop spontaneously under it. Thus, there will be more individual stopped robots under the bigger shelter that will act as "seeds" for new clusters. But we hypothesise that beyond a given size, the shelter will become too big to be chosen. Indeed, the bigger the shelter is, the smaller is the "seed" density under it. This will decrease the probability for a moving robot to encounter a "seed" and form a new cluster with it. Furthermore, we also hypothesise that groups under the smaller shelter would be more packed due to smaller opportunities to move. This will increase stability of aggregates under the smaller shelter by slightly increasing the local density of neighbors. In these ways, the group would be able to choose a shelter not too small (as it is already the case) but also not too big to house the whole population. These last hypotheses will be tested in further simulation works.

We now plan to complete our experimental results by performing choice experiments with more than 10 robots to better tune our simulation tool. Indeed, we also plan to perform simulation experiments in order to find behavioral parameters able to influence this choice. Previous simulation work with this kind aggregation model showed that small modifications of stop and start probabilities can deeply alter aggregation patterns. What is more interesting is that these alterations appear even if only $20 \%$ of the population has modified probabilities [12]. Thus we think that modifying these probabilities can also modify the collective choice, allowing us to control group decisions through the introduction of some modified robots into the population of cockroach-like robots. Moreover, Ame et al. [2] showed that mixing individuals of two different strains of the same species of cockroaches can either lead mixed or segegated groups. According to the affinity between the two strains and the proportion of individuals of each of them, the group can split into two sub-groups. These sub-groups will contain individuals of only one strain and will choose different shelters.

To conclude we argue that this work opens some interesting perspectives for collective robotics. Collective choices could be associated, for instance, with a construction behavior, allowing a group of robots to choose a place to build a "nest" adapted to the size of their population or having some specific environmental properties (for instance light intensity, humidity, etc.). It could also be associated with 
the ordering behavior described in Holland and Melhuish [14], allowing robots to assemble objects of different types in different places. We argue that such associations are new challenges to take up if this collective robotics, based on self-organized mechanisms and/or biologically inspired behaviors, must become an efficient and robust way to achieve complex tasks with groups of numerous small autonomous robots.

\section{REFERENCES}

[1] Agassounon, W., \& Martinoli, A. (2002). A macroscopic model of an aggregation experiment using embodied agents in groups of time-varying sizes. In Proceedings of the 2002 IEEE Systems, Man and Cybernetics Conference, Hammamet, Tunisia. IEEE Press.

[2] Ame, J.-M., Rivault, C., \& Deneubourg, J.-L. (2004). Cockroach aggregation based on strain odour recognition. Animal Behaviour, 68(4), 793-801.

[3] Beckers, R., Holland, O. E., \& Deneubourg, J.-L. (1994). From local actions to global tasks: stigmergy and collective robotics. In Brooks, R., \& Maes, P. (Eds.), Proceedings of the Fourth Workshop on Artificial Life, 181-189, Cambridge, MA. MIT Press.

[4] Bonabeau, E., Dorigo, M., \& Theraulaz, G. (1999). Swarm intelligence : from natural to artificial systems. Oxford University Press, Oxford.

[5] Bonabeau, E., Theraulaz, G., \& Deneubourg, J.-L. (1998). The synchronization of recruitment-based activities in ants. BioSystems, 45, 195-211.

[6] Camazine, S., Deneubourg, J., Franks, N. R., Sneyd, J., Theraulaz, G., \& Bonabeau, E. (2001). Self-organization in biological systems. Princeton University Press, Princeton.

[7] Caprari, G., Estier, T., \& Siegwart, R. (2002). Fascination of down scaling - Alice the sugar cube robot. Journal of Micromechatronics, 1(3), 177-189.

[8] Deneubourg, J. L., Lioni, A., \& Detrain, C. (2002). Dynamics of aggregation and emergence of cooperation. $\mathrm{Bi}$ ological Bulletin, 202(3), 262-7.

[9] Detrain, C., \& Pasteels, J. (1992). Caste polyethism and collective defense in the ant, Pheidole pallidula: the outcome of quantitative differences in recruitment. $\mathrm{Be}$ havioral Ecology and Sociobiology, 29, 405-412.

[10] Dussutour, A., Fourcassié, V., Helbing, D., \& Deneubourg, J. L. (2004). Optimal traffic organization in ants under crowded conditions. Nature, 428(6978), $70-3$.
[11] Franks, N. R., \& Deneubourg, J.-L. (1997). Selforganizing nest construction in ants: the behaviour of individual workers and the properties of the nest's dynamics. Animal Behaviour, 54, 779-796.

[12] Gautrais, J., Jost, C., Jeanson, R., \& Theraulaz, G. (2004). How individual interactions control aggregation patterns in gregarious arthropods. Interaction Studies, 5(2), 245-269.

[13] Grassé, P.-P. (1959). La reconstruction du nid et les coordinations inter-individuelles chez Bellicositermes $\mathrm{Na}$ talensis et Cubitermes sp. La théorie de la stigmergie : essai d'interprétation du comportement des termites constructeurs. Insectes sociaux, 6, 41-81.

[14] Holland, O., \& Melhuish, C. (1999). Stigmergy, selforganisation, and sorting in collective robotics. Artificial Life, 5, 173-202.

[15] Jeanson, R., Blanco, S., Fournier, R., Deneubourg, J. L., Fourcassié, V., \& Theraulaz, G. (2003). A model of animal movements in a bounded space. Journal of Theoretical Biology, 225(4), 443-451.

[16] Jeanson, R., Deneubourg, J., \& Theraulaz, A. G. G. (2004). Modulation of individual behavior and collective decision-making during aggregation site selection by the ant Messor sancta. Behavioral Ecology and Sociobiology, 55, 388-394.

[17] Jeanson, R., Rivault, C., Deneubourg, J.-L., Blanco, S., Fournier, R., Jost, C., \& Theraulaz, G. (2005). Self-organized aggregation in cockroaches. Animal Behaviour, 69(1), 169-180.

[18] Ledoux, A. (1945). Étude experimentale du grégarisme et de l'interattraction sociale chez les Blattidés. Annales des Sciences Naturelles Zoologie et Biologie Animale, 7, 76-103.

[19] Martinoli, A., \& Mondada, F. (1995). Collective and cooperative group behaviours: biologically inspired experiments in robotics. In Khatib, O., \& Salisbury, J. K. (Eds.), Proceedings of the Fourth International Symposium on Experimental Robotics, 3-10, Stanford. Lecture Notes in Control and Information Sciences.

[20] Millor, Pham-Delegue, Deneubourg, \& Camazine (1999). Self-organized defensive behavior in honeybees. Proc Natl Acad Sci U S A, 96(22), 12611-5.

[21] Pasteels, J. M., Deneubourg, J.-L., \& Goss, S. (1987). Self-organization mechanisms in ant societies (i) : trail recruitment to newly discovered food sources. In Pasteels, J. M., \& Deneubourg, J.-L. (Eds.), From individual to collective behavior in social insects, volume 54, 155175, Bâle. Birkhaüser. 
[22] R Development Core Team (2004). R: A language and environment for statistical computing. $\mathrm{R}$ Foundation for Statistical Computing, Vienna, Austria. ISBN 3-90005107-0.

[23] Rust, M. K., Owens, J. M., \& Reierson, D. A. (1995). Understanding and controlling the german cockroach. Oxford University Press, Oxford.

[24] Sahin, E., \& Spears, W. M. (Eds.) (2005). Swarm Robotics, SAB 2004 International Workshop, Santa Monica, CA, USA, July 17, 2004, Revised Selected Papers, volume 3342 of Lecture Notes in Computer Science. Springer.

[25] Seeley, T., Camazine, S., \& Sneyd, J. (1991). Collective decision-making in honey bees: how colonies choose among nectar sources. Behavioural Ecology and Sociobiology, 28, 277-290.

[26] Sugawara, K., \& Sano, M. (1997). Cooperative acceleration of task performance: foraging behavior of interacting multi-robots system. Physica D: Nonlinear Phenomena, 100(3/4), 343-354.

[27] Wagner, I. A., \& Bruckstein, A. M. (2001). Ant robotics. Annals of Mathematics and Artificial Intelligence, 31, 1-238. 